\title{
Heat transfer under high-power heating of liquids. 1. Experiment and inverse algorithm
}

\author{
Sergey B. Rutin ${ }^{\text {a }}$, Aleksandr A. Smotritskiy ${ }^{a}$, Aleksandr A. Starostin ${ }^{\text {a,b }}$, Yuriy S. Okulovsky ${ }^{\text {b }}$ \\ Pavel V. Skripov ${ }^{\mathrm{a}, \mathrm{c}, *}$ \\ a Institute of Thermal Physics, Ural Branch, Russian Academy of Sciences, Amundsena St. 106, Ekaterinburg 620016, Russia \\ ${ }^{\mathrm{b}}$ Ural Federal University named after First President of Russia B.N. Yeltsin, Mira St. 19, 620002 Yekaterinburg, Russia \\ ${ }^{\mathrm{c}}$ Ural Institute of State Fire Service of EMERCOM of Russia, Mira St. 22, 620062 Yekaterinburg, Russia
}

\section{A R T I C L E I N F O}

\section{Article history:}

Received 14 December 2012

Received in revised form 7 February 2013

Accepted 21 February 2013

Available online 22 March 2013

\section{Keywords:}

Pulse heating

Superheated liquids

Thermal conductivity

Heat capacity

Inverse heat conduction problem

Genetic algorithm

\begin{abstract}
A B S T R A C T
A new approach to fluids behavior study in the course of highpower heating has been developed by us. The approach combines experimental method of controlled pulse heating of a wire probe and numerical method of thermophysical properties temperature dependencies recovery from the experimental data. Short (millisecond) characteristic time scale allows working with short-lived fluids, including superheated (with respect to the liquid-vapor equilibrium temperature and/or to the temperature of thermal decomposition onset) ones. Numerical method gives a set of inverse heat conduction problem solutions, based on the results of single pulse experiment. Numerical technique, based on the heat transfer parameters optimization model, is built using genetic algorithms. The approach was applied to saturated hydrocarbons in the temperature range $300-625 \mathrm{~K}$.
\end{abstract}

(c) 2013 Elsevier Ltd. All rights reserved.

\section{Introduction}

To ensure the reliability of equipment involved in the processes of heat transfer, special working fluids, such as advanced energy transmission fluids, high-performance coolants, lubricants, metalworking fluids, etc., are being developed. The informed choice of working fluids and assessment of their durability under conditions of high-power heating require the knowledge of their thermophysical properties over wide ranges of temperatures and characteristic times, including the region of the short-lived states of a fluid. We shall use the term "short-lived" to refer to fluids that lose their stability in the course of heating, namely, superheated (with respect to the liquid-vapor equilibrium temperature and/or the temperature of thermal decomposition onset) liquids. Because of the obvious experimental difficulties, the properties of such liquids remain poorly known so far [1-3].

The properties of fluids are normally measured under conditions of small temperature perturbations. Both stationary and non-stationary measurement techniques have been developed, with the transient hot-wire method being most prevalent [4-8].

\footnotetext{
* Corresponding author at: Institute of Thermal Physics, Ural Branch, Russian Academy of Sciences, Amundsena St. 106, Ekaterinburg 620016, Russia. Tel.: +7 343 3745442; fax: +7 3432678800 .

E-mail address: pavel-skripov@bk.ru (P.V. Skripov).
}

Thermophysical properties are determined from the measured time evolution of the probe temperature $\Delta T(t)$ using analytical linear approximation. This approximation assumes temperatureindependent properties over the experimental $\Delta T$ range. In modern versions of the transient hot wire method [7,8], analytical approximation is replaced with detailed, spatially- and time-resolved numerical modeling of heat exchange.

For the analysis of thermal processes involving rapid, highpower heat release, the validity of conventional linear approximation deteriorates. This motivated the development of the probe pulse heating method combined with numerical simulation of heat transfer parameters based on the experimental results. The key feature of our approach is electronic control of the probe heating power against a background of the thermophysical properties variations with the temperature of both probe and substance [9-11]. An advanced variant of the electronic control system for quasi-static conditions (with respect to the temperature) is described elsewhere [12].

In the experiments, weight-average probe temperature $T(t)$ for a given heating power history $P(t)$ (where $t$ is the time) is recorded. Differences in the heating curves under the same heating scenarios for different samples or different pressures for the same sample reflect differences in the thermophysical properties. These data are sufficient to calculate the density of heat flux, which is an integral characteristic of the pulse experiment. 
Experimental results provide sufficient information to test and to validate the chosen heat transfer model for the probe-liquid system. The analytical linear model based on assumption of properties constancy is no longer valid: wide variations of temperature require taking into account properties' temperature dependencies. Consequently, full transient heat transfer problem needs to be solved numerically to describe the system.

The predicted $T(t)$ values are obtained from the direct numerical solution of transient heat transfer problem for the system using the finite volume method. Estimation of heat transfer parameters is performed by comparing the numerical solution of the direct problem and the experimental data. Generally, evaluation of parameters for the model with known structure is provided by various optimization procedures [13-15]. Complexity of the problem required selection of the robust, gradient-free optimization method. Therefore, solution of inverse heat conduction problem (IHCP) based on the genetic algorithm (GA) method was chosen. In this approach, the two-dimensional heat transfer problem was considered; the solutions (temperature-dependent thermophysical properties) were approximated with polynomial functions of the second order. The results for the saturated hydrocarbons in the temperature range of $20-350{ }^{\circ} \mathrm{C}$ are presented as an example.

\section{Theoretical background}

Optimization methods have recently become widespread due to the development of computer technology. These procedures are implemented for IHCP solution along with classical analytical methods. Much attention in the literature is devoted to onedimensional IHCP $[14,15]$.

GA provides an efficient search for solutions of different types of problems [13], including IHCP [16-21], and has shown superior performance in locating global optima for multidimensional problems.

One of the key GA features is a robust operation in a course of a multi-parameter optimization by mimicking the biological evolution. During the evolution of the population, a sequence of generations is created. GA assures survival and propagation of the best entities in the new generation.

\section{Experimental}

Experimental results are obtained using a constant heating power mode $[9,10]$. The method implements electrical power control directly during the pulse. The power supplied to the probe is proportional to the driving voltage: $P(t)=n \cdot U(t)$, where $n$ is a constant factor. The control procedure is based on the ProportionalIntegral (PI) controller implementation covered by the negative feedback [22]. Generally, the driving voltage parameters can be chosen arbitrarily. Based on our experience, a constant heating power mode generally works well for determination of thermophysical properties. Repeatability level, obtained in a series of pulses, was $0.05 \%$ [9]. Schematic diagram of the home-made experimental setup is shown on Fig. 1.

Experimental setup operates as follows. A sync pulse generator forms both clock pulse and a single input pulse of a certain amplitude and length. As clock pulse is generated, analog-digital converter (ADC) (3) is switched on, and the power control unit (6) is shifted to an active mode. As a result, current flow appears in a probe (7) - standard resistor (5) circuit. Voltage drop across the probe and standard resistor is applied to PI-power controller (2) input. Simultaneously, the same voltage drop is recorded by ADC (3) and transferred to a PC (4).

Platinum wire probe has a diameter of $20 \mu \mathrm{m}$ and length of $1, \ldots, 2 \mathrm{~cm}$. As usual [3-12], probe combines functions of both a

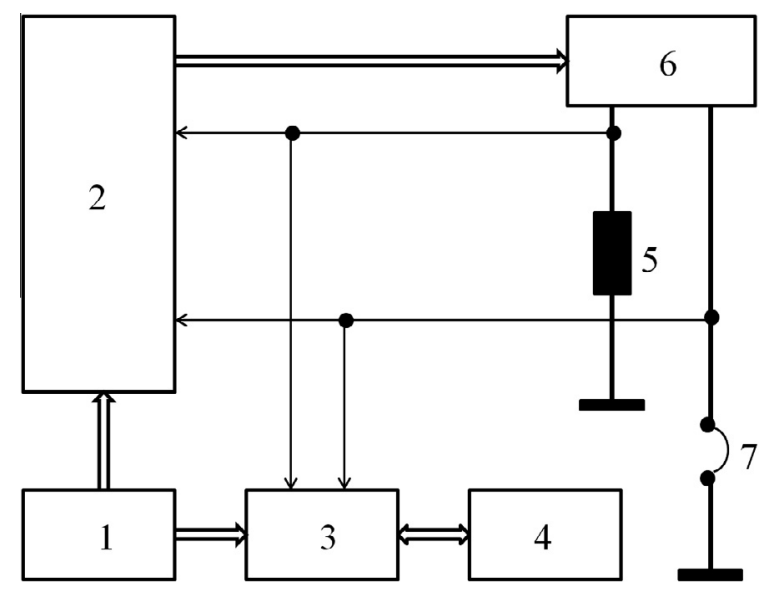

Fig. 1. Schematic diagram of the experimental setup. 1 - Control pulse generator; 2 - PI-power controller; 3 - analog-digital converter (ADC); 4 - computer; 5 - precise standard resistor; 6 - power control unit; 7 - wire probe $\left(R_{w}\right)$, connected with the standard resistor $\left(R_{i}\right)$ in series.

heater and a resistance thermometer. Standard reference table for platinum thermometers is implemented for resistance-to-temperature conversion. A relative version of the method was applied. Probe calibration verification is carried out by the temperature of attainable superheat measurements [1-3] for saturated hydrocarbons at a pressure $0.95 p_{c}$, where $p_{c}$ is the thermodynamic critical pressure.

Primary values are the voltage drop across the probe $U_{w}(t)$ and the standard resistor $U_{i}(t)$. Using these values, current flow through the probe $I(t)$, power $P(t)$, probe resistance $R(t)$ and weightaveraged probe temperature $T(t)$ are calculated. Voltage drops $U_{w}(t)$ and $U_{i}(t)$ are recorded via ADC L-card, E20-10, Rev B (http://lcard.ru). Basic ADC characteristics are as follows: signalto-noise ratio is $-73 \mathrm{db}$, analog signal bandwidth is $1.25 \mathrm{MHz}$, resolution is 14 bits, and sampling rate is $10 \mathrm{MHz}$. Measurement uncertainty is $0.2 \%$. A recorded data array contains $10^{3}-10^{5}$ samples, sufficient for post-processing. LCR-meter (LCR-819, http://www.instek.cn/en/brand.aspx) was used for resistance measurement both for the standard resistor and for the probe at initial temperature. Measurement uncertainty for LCR-819 is $0.05 \%$.

Mercury laboratory thermometer with the scale interval of $0.1{ }^{\circ} \mathrm{C}$ was applied for the measurement of the initial temperature in a substance bulk, $T_{0}$. Taking into account the residual temperature gradients in the working cell (Fig. 2), the uncertainty of this measurement is estimated to be $0.05 \%$.

Measurement uncertainties of $U_{w}(t)$ and $U_{i}(t)$ are derived from the ADC uncertainty combined with the scaling amplifiers errors and make $0.3 \%$ total. $I(t)$ measurement uncertainty includes the same components as well as standard resistor $R_{i}$ value measurement error and makes $0.35 \%$ total. Power $P(t)$ and probe resistance $R(t)$ calculation uncertainties are equal and both derived from the $U_{w}(t)$ and $I(t)$ uncertainties, yielding $0.65 \%$.

Uncertainty of calculation of the weight-average probe temperature $T(t)$ is derived from the same values together with:

- probe resistance at initial temperature $R_{0}$ measurement error (0.05\%);

- initial temperature in a substance bulk $T_{0}$ measurement error (0.05\%);

- resistance-to-temperature conversion error $(0.2 \%)$.

As a result, measurement uncertainty of the weight-average probe temperature is estimated to be $1.0 \%$.

Another source of uncertainty arises from the signal noise in the setup electronic components. Noise spectrum is flat random over 


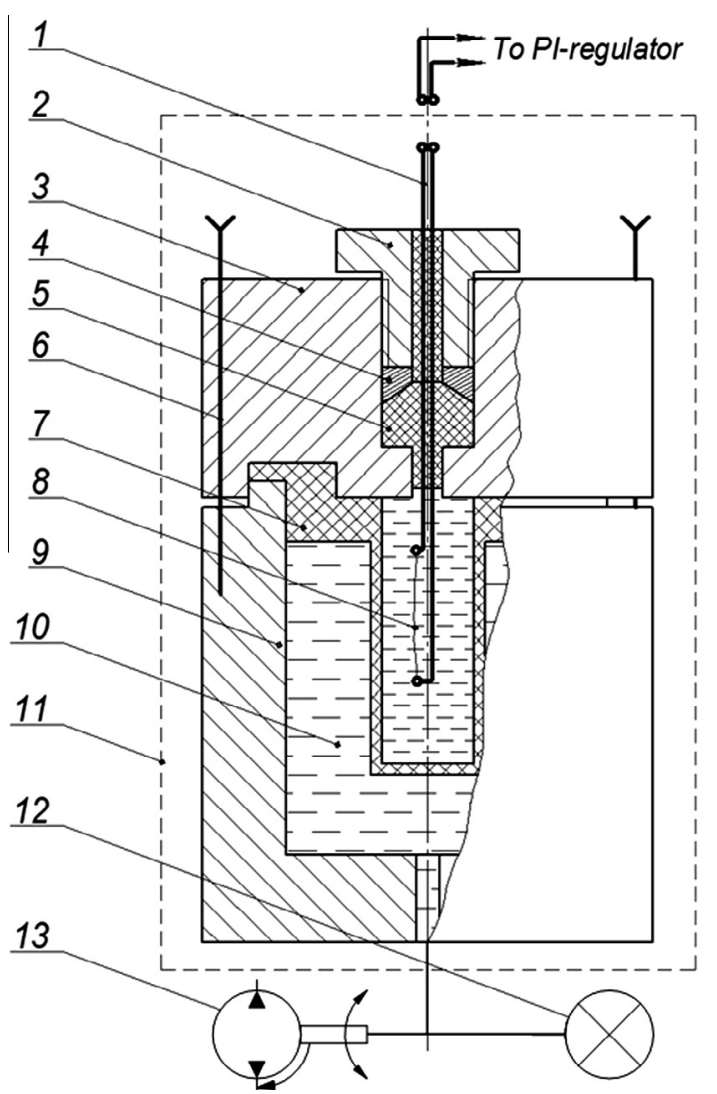

Fig. 2. Schematic of the pressure cell: 1 - electrical lead through, 2 - sealing nut, 3 top flange, 4 - spacer, 5 - sealing, 6 - pressurezation bolts, 7 - teflon vessel with the substance under investigation, 8 - wire probe, 9 - pressure chamber, 10 - pressuretransmitting medium, 11 - thermostat, 12 - pressure sensor, 13 - compression unit

the entire frequency range. As a result, data array is smoothed using the cubic spline [9]. This procedure can significantly improve the sensitivity of the method, without loss of information in the recorded data. Finally, the uncertainty due to the signal noise is proved to be much smaller than other components and can be ignored after smoothing.

\section{Modeling}

Direct solution of heat transfer problem refers to determination of the transient temperature field from the known thermophysical properties. Mathematical model described in detail in Ref. [19] and reproduces the conditions of the pulse experiment. Schematic of the probe assembly and computational model is shown on Fig. 3. Heat-balance equation with axial symmetry was chosen as a model:

$c_{p}(T) \rho(T) \frac{\partial T}{\partial t}=\frac{1}{r} \frac{\partial}{\partial r}\left[r \lambda(T) \frac{\partial T}{\partial r}\right]+\frac{\partial}{\partial z}\left[\lambda(T) \frac{\partial T}{\partial z}\right]+q_{v}(t)$

In the above equation, $c_{p}$ is the specific isobaric heat capacity, $\lambda$ is the thermal conductivity, $\rho$ is the density, $q_{v}$ is the heat release power, $t$ is the time, $z$ is the axial coordinate, and $r$ is the radial coordinate.

Interface boundary conditions are:

$-\left.\lambda_{f}(T) \frac{\partial T}{\partial r}\right|_{r=r_{0}}=-\left.\lambda_{w}(T) \frac{\partial T}{\partial r}\right|_{r=r_{0}}$

$\left.T_{f}\right|_{r=r_{0}}=\left.T_{w}\right|_{r=r_{0}}$

$\left.T\right|_{r \leqslant r_{0} ; t=0}=T_{0}$
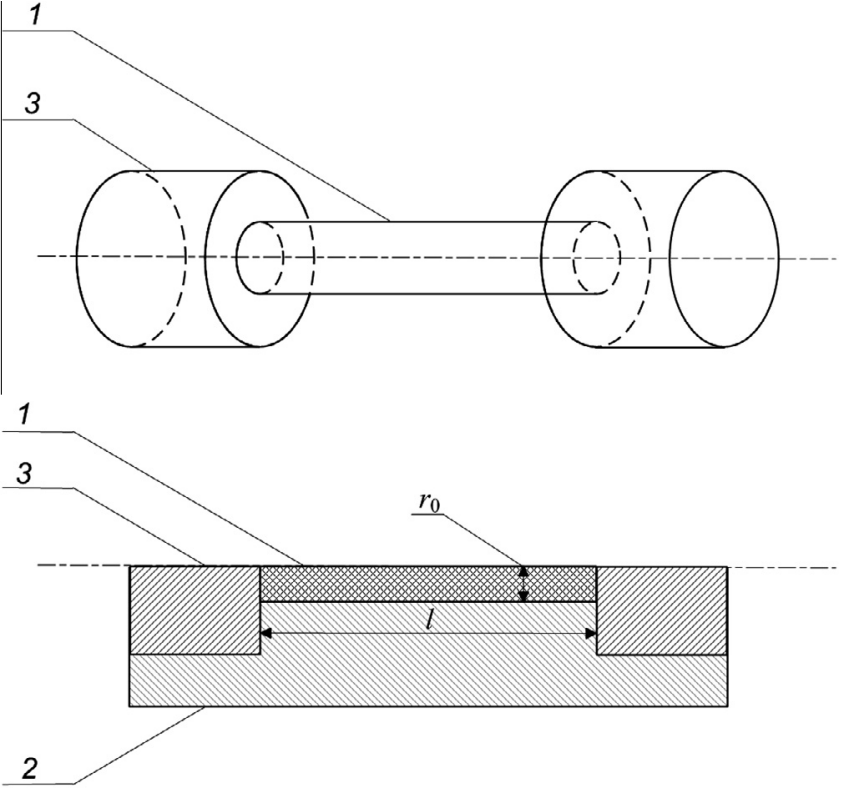

Fig. 3. Schematic of the probe assembly and computational model: 1 - wire probe 2 - substance under investigation, 3 - copper lead wire, soldered to the probe, $l$ probe length, $r_{0}$ - probe radius.

where $r_{0}$ is the radius of the probe, and the subscripts $f$ and $w$ refer to the properties of fluid and probe, respectively.

The predicted temperature histories are obtained from the direct numerical solution of transient heat transfer problem for the system. Solution is performed by time integration of Eq. (1) using finite volume method. Discretization was chosen to provide a balance between accuracy and CPU time consumption [19]. CPU time, consumed for 1 direct solution, is $10 \mathrm{~s}$.

The input data are:

- initial temperature $T_{0}$;

- probe parameters $\left(l, r_{0}\right)$;

- thermophysical properties' temperature dependencies of the probe material, current leads and the liquid;

- pulse length and electrical power.

Regular set of assumptions is considered:

- liquid is quiescent;

- Fourier hypothesis holds for every point of the system;

- heat transfer proceeds only via thermal conductivity;

- cylindrical symmetry;

- probe edges and the inner wall of the pressure vessel are held at a constant temperature, and the temperature field is uniform at the initial time.

Transient temperature fields for the probe and liquid, and heat flow evolution on the probe-liquid boundary are obtained in the course of numerical solution. Method performance is demonstrated by comparing the experimental and the predicted temperature histories for two cases. Fig. 4a shows the case when constant and linearly dependent thermophysical properties are assumed. Fig. $4 \mathrm{~b}$ shows a comparison of experimental results and predictions obtained using temperature-dependent thermophysical properties. Difference between the experimental and the predicted temperature curves for the first case (Fig. 4a) is about $10 \%$, for the second case (Fig. 4b) curves coincide within the scatter of experimental data. Thus, effect of the thermophysical properties temperature dependence is shown. 


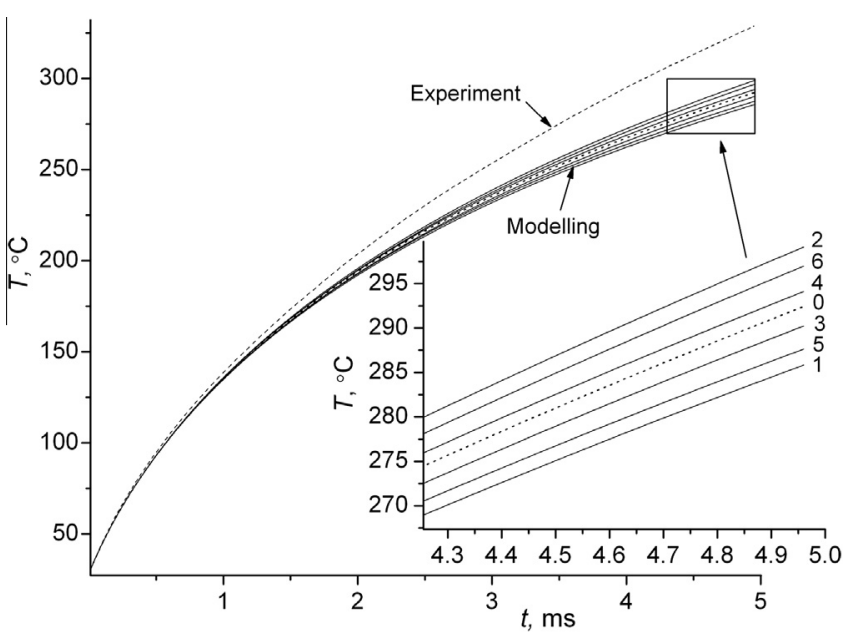

Fig. 4a. Numerical modeling of the probe temperature evolution for $n$-hexadecane under constant heating power mode. Lines $1, \ldots, 6$ demonstrate the effect of temperature dependence of thermophysical properties. Linear temperature dependence was assumed in all cases. Line markings: 0 - baseline (no temperature dependence, $\left.\lambda\left(T_{\max }\right)=\lambda\left(T_{0}\right)=\lambda_{0} ; \quad \rho c_{p}\left(T_{\max }\right)=\rho c_{p 0}\right) ; 1 \quad-\left(T_{\max }\right)=1.1 \lambda_{0} ; 2 \quad-$ $\lambda\left(T_{\max }\right)=0.9 \lambda_{0} ; 3-\rho c_{p}\left(T_{\max }\right)=1.1 \rho c_{p 0} ; 4-\rho c_{p}\left(T_{\max }\right)=0.9 \rho c_{p 0}$. Lines 5 and 6 are the combinations of lines $1 \& 4$ and $2 \& 3$, respectively.

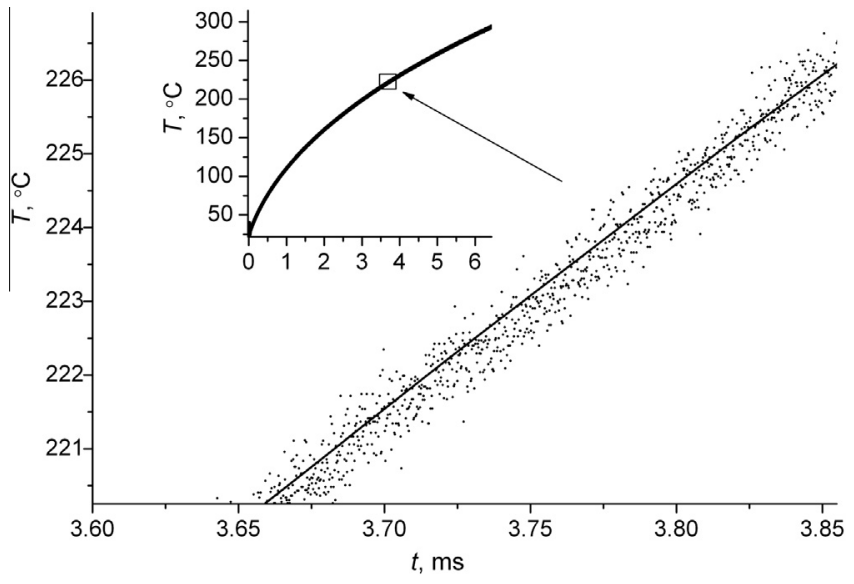

Fig. 4b. Experimental and calculated temperature histories for $n$-hexane. Dots corresponds to the raw experimental temperature curve. Solid line corresponds to predicted temperature curve. Insert shows both curves in a full scale.

IHCP solution implies determination of thermophysical properties from the experimental heating curves. Thermophysical properties evaluation is implemented in optimization procedure based on GA, see Fig. 5 .

A set of model parameters is optimized to minimize the deviation between the measured and predicted temperature histories. Thermophysical properties at initial temperatures are assumed to be known a priori. Thermophysical properties temperature dependencies are approximated with the polynomial functions of the second order:

$\lambda(T)=a_{0}+a_{1} \cdot T+a_{2} T^{2} \quad \rho c_{p}(T)=b_{0}+b_{1} \cdot T+b_{2} T^{2}$

The following model parameters are used: thermal conductivity and volumetric heat capacity temperature dependencies, probe length and diameter, and a value of specific heating power. Consequently, each entity $(e)$ is characterized by eight parameters:

$e=\left[a_{0}, a_{1}, a_{2}, b_{0}, b_{1}, b_{2}, r, l\right]$

GA population is formed by 30 entities. The values of parameters are picked up randomly within the specified range. Problem solution is encoded with a set of a floating-point numbers. Floating-point number implementation instead of a binary string allows increasing algorithm performance. Solution selection is based on the values of objective function. Objective function to be minimized, $F_{c}$, is inversely proportional to the standard deviation between the measured and predicted temperatures. $F_{c}$ value varies between 0 and $1 . F_{c}$ is written as follows:

$$
F_{c}=\frac{k}{k+\sqrt{\frac{1}{n} \cdot \sum_{i=1}^{n}\left(T^{\prime}\left(t_{i}\right)-T\left(t_{i}\right)\right)^{2}}}
$$

where

- $T^{\prime}\left(x_{i}\right)$ is the probe temperature obtained from the solution of the direct problem, at the instant $x_{i}$;

- $T\left(x_{i}\right)$ is the probe temperature observed in the experiment;

$-k>1$ is a scaling factor, generally its value is set equal to 10 .

For algorithm accuracy enhancement, the following heuristic methods are adopted:

- gene mutation area increases with the objective function reduction;

- the higher gene objective function is, the smaller are the search steps;

- gene mutation area decreases with time, what corresponds to the method of simulated annealing.

Optimization procedure, based on the GA, is implemented in the in-house software. Algorithm testing has revealed that solutions with discrepancy less than $1 \%$ between the experimental and predicted temperature data are obtained after about ten GA generations for the reference substances.

\section{Calibration procedure}

The common problem with the analysis of pulse heating experiments is the uncertainty in the probe size that is not easily quantifiable under given wire manufacturing and mounting techniques; the uncertainty in wire diameter can be up to $10 \%$. Conventional solution of this problem is probe calibration using reference substances [6]. Generally, saturated hydrocarbons, such as $n$-hexadecane and $n$-hexane are used as calibration fluids. To find the effective values of the wire diameter and length, an iterative procedure described above is applied, see Fig. 6. Length and diameter of the wire are formally defined in a course of calibration, according to the accepted thermophysical model. Mathematical model simplifications are included in the results of the calibration, and the resulting fitted values are effective parameters that also compensate for the model assumptions. All subsequent measurements using the calibrated probe are relative.

In our work, calibration procedure is also performed for each probe. Uncertainty of the wire diameter is $1-2 \mu \mathrm{m}$, or $5 \%-10 \%$. Probe length can be estimated by the value of the electrical resistance. To find effective values of the wire diameter and length, an iterative procedure described above is applied. In this case, only probe geometry is optimized; the other model parameters are assumed to be known and not included in the optimization procedure.

An example of calibration procedure implementation for probe diameter refinement is shown on Fig. 7. Shown are calculated temperature histories, corresponding to a set of probe diameters: optimized probe diameter as well diameter, that differs from the refined one by $1 \%$. In such a way, features of numerical model used are taken into account during calibration procedure.

Total method uncertainty is composed of the following components. Uncertainty of experimental measurement of the probe 


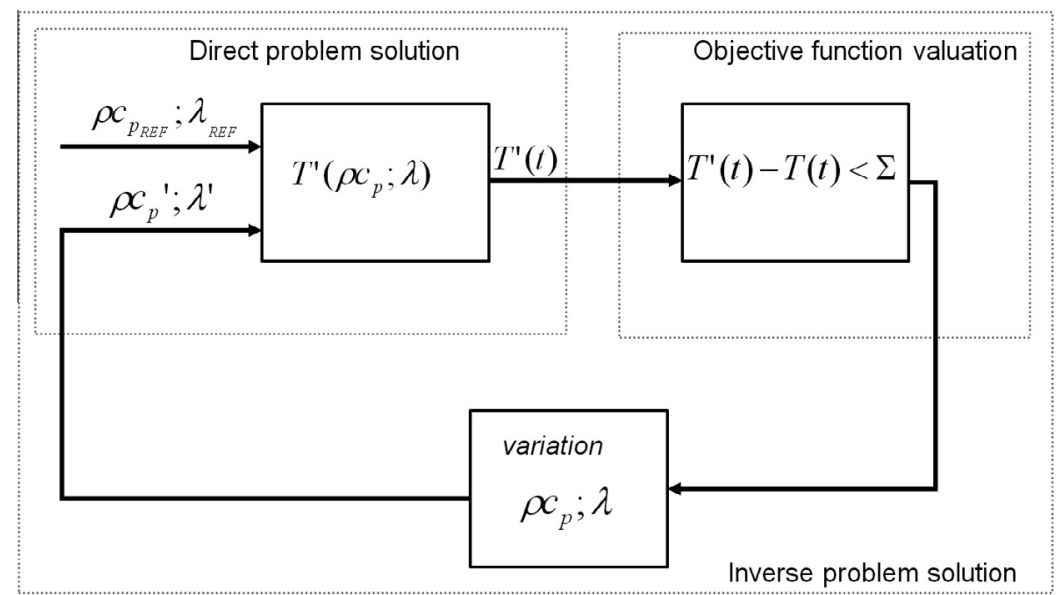

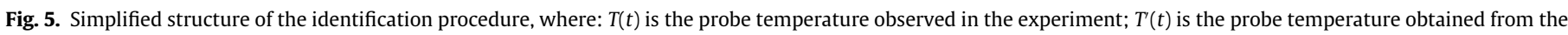

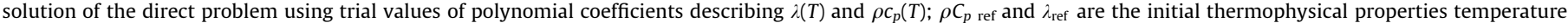

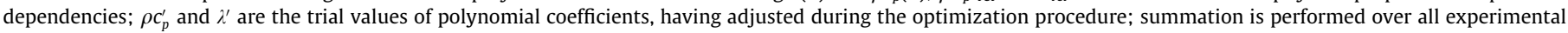
points ( $n$ total).

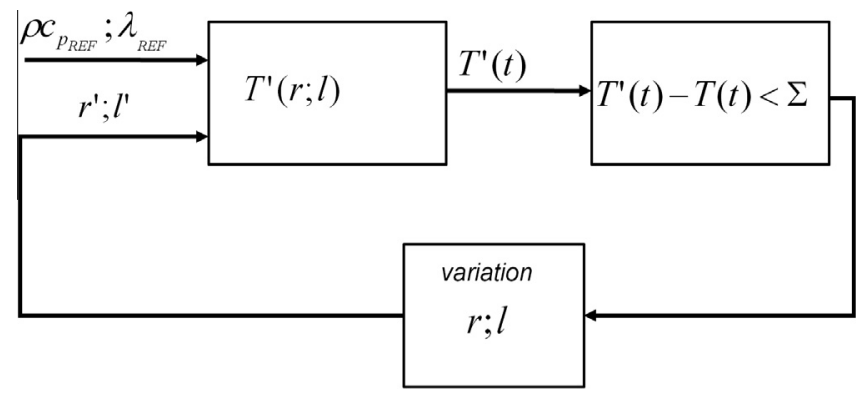

Fig. 6. Structure of the calibration procedure, where $r$ and $l$ refer to initial values of the wire diameter and length, respectively; $r^{\prime}$ and $l^{\prime}$ refer to values of the wire diameter and length, refined in the course of calibration procedure.

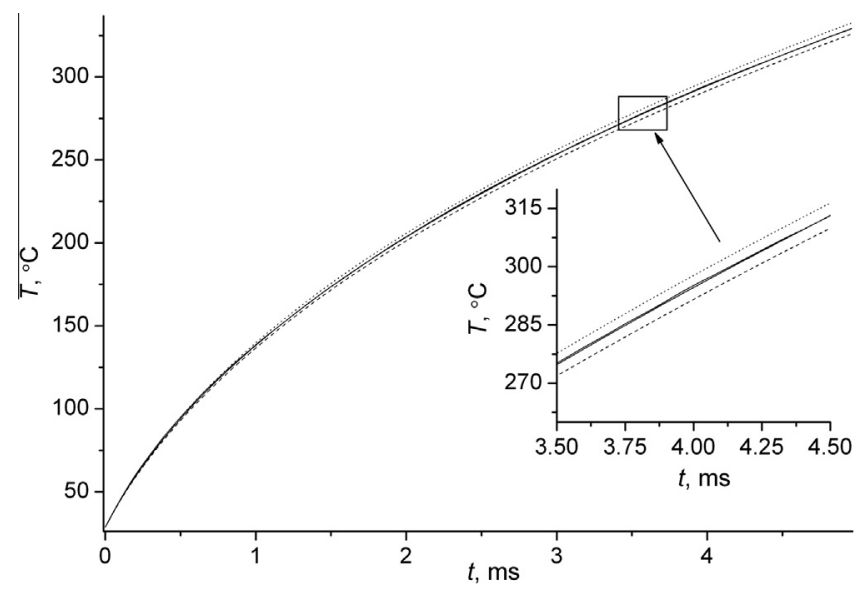

Fig. 7. An example of calibration procedure implementation for the probe diameter refinement. The difference between the calculated temperature histories for a set of probe diameters (refined diameter and diameters, differs from refined by $\pm 1 \%$ ) is shown. The upper curve corresponds to the $1.01 d_{0}$, the lower curve corresponds to $0.99 d_{0}$.

temperature, numerical model errors, and inverse problem solutions scatter.

Total uncertainty is evaluated for the temperature range $300-$ $580 \mathrm{~K}$ for the reference substances. $\rho c_{p}$ uncertainty is $\pm 2 \%$ and $\lambda_{\text {ref }}$ uncertainty is $\pm 3 \%$. Instrument uncertainty for the cases of wider temperature ranges and should be discussed separately.

\section{Results and discussion}

The approach for the thermophysical properties temperature dependence evaluation is demonstrated using a set of saturated hydrocarbons (as the reference fluids). Fig. 8 compares the results obtained in this work and available data for the thermophysical properties of $n$-tetradecane. Ten solutions with the best value of objective function are presented. Discrepancy of monitored parameter (a deviation between smoothed experimental and calculated thermograms) is in the range from $0.1 \%$ to $0.2 \%$. Despite the difference in the experimental conditions, there is a consistency of reference data with the results of this work. Constant heating power mode provides identity of the heat release conditions for a set of specimens with different thermophysical properties.

Accuracy of heuristic optimization method was evaluated in the numerical experiment. A bank of solutions was generated, data from [23-29] were used as the reference ones. Fig. 9 shows objective function dependence on the number of iterations for the two independent sets of solutions. The objective function scatter, which is a qualitative criterion of computational model and system described conformity, is about $7 \%$ when the number of iterations is about 50 or more.

In conventional GA the best solution propagates to the next generation. Iteration procedure, adopted here, has a number of features. Stopping criterion for the iterative process is reached, if there is no change in the best solutions during several iterations. After that, all entities from the current population are placed in the archive. Subsequent new solutions are generated by applying genetic operators to the solutions from the archive, to refine the identified solutions more efficiently. As a result, objective function increases with generations sometimes, see Fig. 9.

One of actual problems of the method being discussed is striking a balance between increasing the heating rate in the course of experiment and experiment sensitivity. The higher heating rate, the lower is the difference between the weight-averaged and maximum over the volume probe temperatures. Thus, the "end" of the amendments is less important. On the other hand, thickness of the heated layer increases with the experiment duration. This leads to an increase in sensitivity and information content of the experiment.

To solve this problem, both heating rate and experiment duration are chosen carefully. The use of different diameter probes is also suggested. 

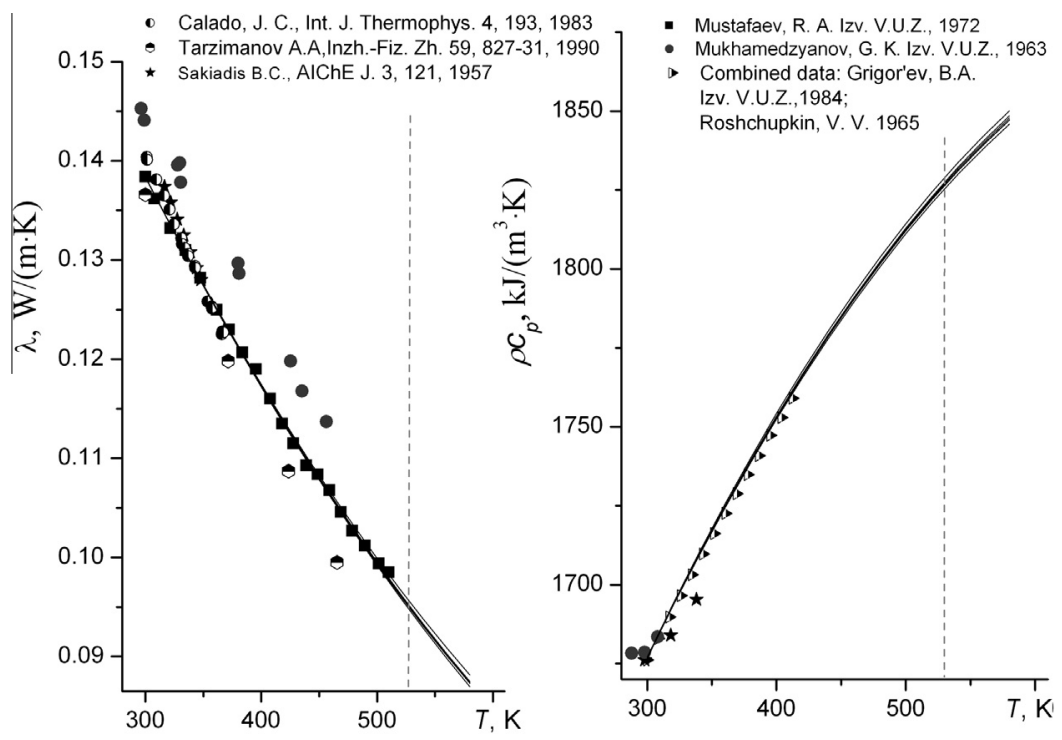

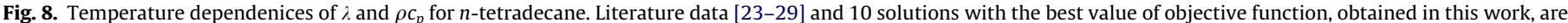
shown. The vertical line is the liquid-vapor equilibrium temperature at atmospheric pressure.

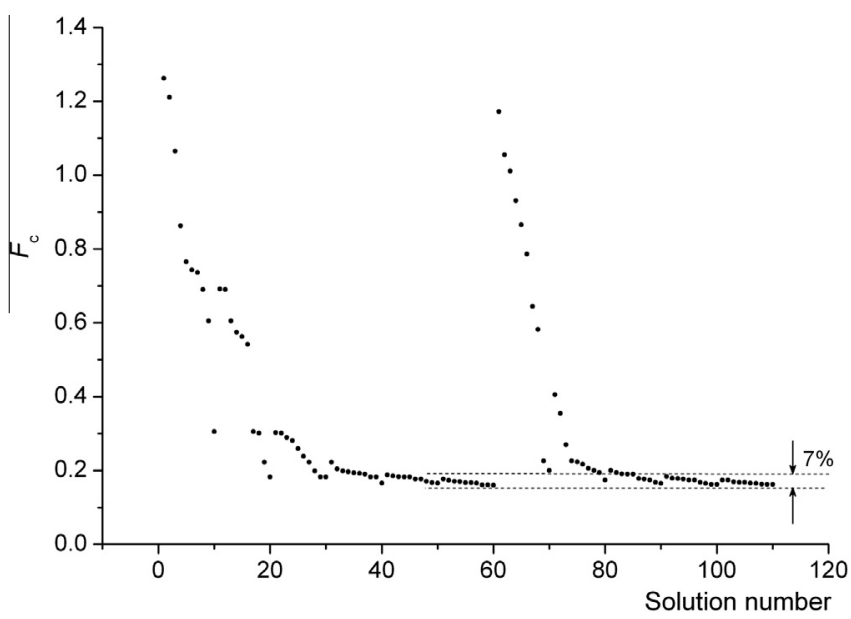

Fig. 9. Objective function dependence on the number of iterations for the two independent sets of solutions.

\section{Conclusions}

We are developing an efficient method for thermophysical property investigation. Method allows evaluating the temperature dependence of the properties of the studied substance in a single pulse experiment. The experimental setup allows operating on the millisecond time scale and accessing the region of short-lived states of substance. Functionality and sensitivity of the method was tested during the calibration procedures. In the course of the experiments and numerical simulation, the conformed data were obtained for saturated hydrocarbons.

A number of problems is to be solved in the future work. At this stage, the method assumes working with substances for which initial data are available. "Binding" of the data to the thermophysical properties at the initial temperature $T_{0}$ is required. In order to form such a pool of data at $T_{0}$, the combined use of our method and one of the classic experimental methods is accepted. The main opportunity to improve the performance of the method is the reduction of the impact of experimental errors and numerical simulation on the results. Further work will focus on the choice of hardware and software to minimize errors in the identification of the object.
Another direction of this research is the use of different modes of pulse heating of the probe and comparing the results with the results obtained at a constant heat release power. To increase the information content of the experimental heating curves, we are developing the methods of controlled pulse heating, which makes it possible to increase the scale of the useful signal obtained in experiment. Sequence of heating pulses mode gives additional possibilities for experimenter. The alternation of short (1 $\mathrm{ms}$ in order of magnitude) high-power pulses of heating and short pauses between pulses enhances the resolving power of experiment, as it was revealed by our experience.

\section{Acknowledgments}

The study was partially supported by RFBR, project Nos. 10-08-00538-a, 12-08-31134, by CRDF-UrB RAS, project No. RUE1-7033-EK-11. We gratefully acknowledge Dr. Andrei Kazakov (NIST, Boulder) for his helpful consultations.

\section{References}

[1] V.P. Skripov, Metastable states, J. Non-Equilib. Thermodyn. 17 (1992) 193-236

[2] P.V. Skripov, A.P. Skripov, The phenomenon of superheat of liquids: in memory of Vladimir P. Skripov, Int. J. Thermophys. 31 (4-5) (2010) 816-830.

[3] E.D. Nikitin, The critical properties of thermally unstable substances: measurement methods, some results and correlations, High Temp. 36 (1998) 305-318.

[4] M.J. Assael, K.D. Antoniadis, W.A. Wakeham, Historical evolution of the transient hot-wire technique, Int. J. Thermophys. 31 (2010) 1051-1072.

[5] R. Greger, H.J. Rath, Measurement of the thermal conductivity of fluids with low viscosity under reduced gravity conditions using the transient hot-wire technique, Int. J. Heat Mass Transfer 38 (6) (1995) 1105-1110.

[6] H.M. Roder, A transient hot-wire thermal conductivity apparatus for fluids, J. Res. Natl. Inst. Stand. Technol. 86 (5) (1981) 457-480.

[7] M.J. Assael, L. Karagiannidis, N. Malamataris, W.A. Wakeham, The transient hot-wire technique: a numerical approach, Int. J. Thermophys. 19 (2) (1998) 379-389.

[8] H. Xie, H. Gu, M. Fujii, X. Zhang, Short hot wire technique for measuring thermal conductivity and thermal diffusivity of various materials, Meas. Sci. Technol. 17 (2006) 208-214.

[9] S.B. Rutin, P.V. Skripov, Apparatus for studying heat transfer in nanofluids under high-power heating, J. Eng. Thermophys. 21 (2) (2012) 144-153.

[10] S.B. Rutin, P.V. Skripov, Heat transfer in supercritical fluids under pulse heating regime, Int. J. Heat Mass Transfer 57 (1) (2013) 126-130.

[11] P.V. Skripov, A.A. Smotritskiy, A.A. Starostin, A.V. Shishkin, A method of controlled pulse heating: applications, J. Eng. Thermophys. 16 (3) (2007) 155163. 
[12] S.A. Zhukov, S.Yu. Afanas'ev, S.B. Echmaev, Concerning the magnitude of the maximum heat flux and the mechanisms of superintensive bubble boiling, Int. J. Heat Mass Transfer 46 (18) (2003) 3411-3427.

[13] L. Davis, Handbook of Genetic Algorithms, Van Nostrand Reinhold, NY, 1991

[14] J.V. Beck, B. Blackwell, C.R. St, Clair, Inverse Heat Conduction - Ill Posed Problems, Wiley-Interscience, NY, 1985.

[15] O.M. Alifanov, Inverse Heat Transfer Problems, Springer-Verlag, Berlin, 1994

16] M. Raudensky, K.A. Woodbury, J. Kral, T. Brezina, Genetic algorithm in solution of inverse heat conduction problems, Numer. Heat Transfer B (28) (1995) 293 306

[17] B. Czel, G. Grof, Genetic algorithm-based method for determination of temperature-dependent thermophysical properties, Int. J. Thermophys. 30 (6) (2009) 1975-1991.

[18] S. Garcia, J. Guynn, E.P. Scott, Use of genetic algorithms in thermal property estimation: Part II - Simultaneous estimation of thermal properties, Numer. Heat Transfer A 33 (1998) 149-168.

[19] V.I. Gorbatov, Yu.S. Okulovskii, P.V. Skripov, A.A. Smotritskiy, A.A. Starostin, Identification of heat-exchange parameters under intensive pulse heating of a wire in a fluid, J. Eng. Thermophys. 19 (3) (2010) 144-153.

[20] B. Czel, G. Grof, L. Kiss, Simultaneous measurement of temperature dependent thermophysical properties, Jpn. J. Appl. Phys. 50 (2011). 11RE05.
[21] B. Czél, G. Gróf, Inverse identification of temperature-dependent thermal conductivity via genetic algorithm with cost function-based rearrangement of genes, Int. J. Heat Mass Transfer 55 (15-16) (2012) 4254-4263.

[22] P. Horowitz, W. Hill, Art of Electronics, second ed., Cambridge University Press, New York, Melbourne, 1989 (Chapter 6.24)

[23] J.C.G. Calado, J.M.N.A. Fareleira, C.A. Nieto de Castro, W.A. Wakeham, Thermal conductivity of five hydrocarbons along the saturation line, Int. J. Thermophys. 4 (1983) 193.

[24] B.C. Sakiadis, J. Coates, Studies of thermal conductivity of liquids: Part III, AIChE J. 3 (1) (1957) 121-126.

[25] A.A. Tarzimanov, R.A. Sharafutdinov, F.R. Gabitov, F.D. Uzmuchmedov, Inzh.Fiz. Zh. 59 (1990) 827-831 (in Russian).

[26] G.K. Mukhamedzyanov, A.G. Usmanov, A.A. Tarzimanov, Izv. Vyssh. Uchebn. Zaved. Neft Gaz. 9 (1963) 75-79 (in Russian).

[27] B.A. Grigor'ev, R. A Andolenko, Izv. Vyssh. Uchebn. Zaved. Neft Gaz. 27 (2) (1984) 60 (in Russian).

[28] V.V. Roshchupkin, Nek. Vopr. Fiz. Tekh. Yad. Reakt. (1965) 151-153 (in Russian).

[29] R.A. Mustafaev, Teplofiz. Vys. Temp. 12 (1974) 883-886 (in Russian). 